\title{
Primary Inflammatory demyelinating pseudotumor in the left frontal lobe with meningeal involvement presenting as malignant neoplasms
}

\author{
Yuyuan Ma', Hong Mou'², Xueqiang $\mathrm{Cai}^{3}$, Jie Ma² and Gang Lư ${ }^{*}$
}

\begin{abstract}
Background: Tumor-like demyelinating lesion is a rare form of IP, often indistinguishable from brain tumors on CT or MR imaging.

Case presentation: We report here a case of pathologically confirmed tumor-like demyelinating lesions. The clinical manifestations, image feature and pathology of this case are also discussed together with a review of the relevant literature.

Conclusion: The correct diagnosis of tumor-like demyelinating lesion mainly depends on the postsurgical histopathologic examinations. We suggest complete resection of the intracranial lesion and involved dura, followed by administration of short term oral prednisolone therapy will be good for preventing recurrence.
\end{abstract}

Keywords: Inflammatory pseudotumors, Demyelinating lesion, Intracranial, MRI, Steroid therapy

\section{Background}

Inflammatory pseudotumor (IP) belongs to heterogenous disease with unclear pathogenesis and nomenclature. It was first described in the lung as a lesion of plasma cell granuloma in 1973 by Bahadori and Liebow [1]. Intracranial involvement of IP was not reported until 1980 [2]. The central nervous system is extremely rare to be invaded primarily, only fewer than 80 cases have been described [3-5]. The occurrence of this lesion in intracranial location is still infrequent. Tumor-like demyelinating lesion is a rare form of IP, which is hard to be distinguished from brain tumors by CT or MR imaging [6]. Here, we report a case of pathologically confirmed tumorlike demyelinating lesion. The clinical manifestations, image features and pathology of this case are also discussed together with a review of the relevant literature.

\footnotetext{
* Correspondence: lugang85893451@163.com

'Department of Neurosurgery, Zhejiang Provincial People's Hospital,

Hangzhou, Zhejiang 310014, China

Full list of author information is available at the end of the article
}

\section{Case presentation}

In March 2013, a 54-year-old woman came for us because of a partial seizure attack, her chief complaints are nausea and left frontal headaches for 2 months. She had no previous history of illness except a gastric ulceration for 5 years. Physical examination was negative. The routing laboratory investigations were within normal reference ranges. Contrast-enhanced CT scan showed a heterogeneously enhancing mass lesion in the left frontal lobe with mass effect and significant surrounding edema. And the lesion had mixed higher signal on both $\mathrm{T} 2$ and $\mathrm{T} 1$ weighted MRI. The signal got markedly higher on FLAIR, and severe brain edema was noticed too (Fig. 1a, b, c). Contrast-enhanced MRI showed a heterogeneous enhancement lesion, which is dural-based, ill-defined and infiltrative to the surrounding brain parenchyma (Fig. 1d). The dural enhancement extended into not only the frontal base, but also the top of orbit. Firstly, the lesion was considered as a malignant neoplasm. The differential diagnosis could be high-grade glioma or anaplastic meningioma. Then, the patient underwent frontal craniotomy in neurosurgical 

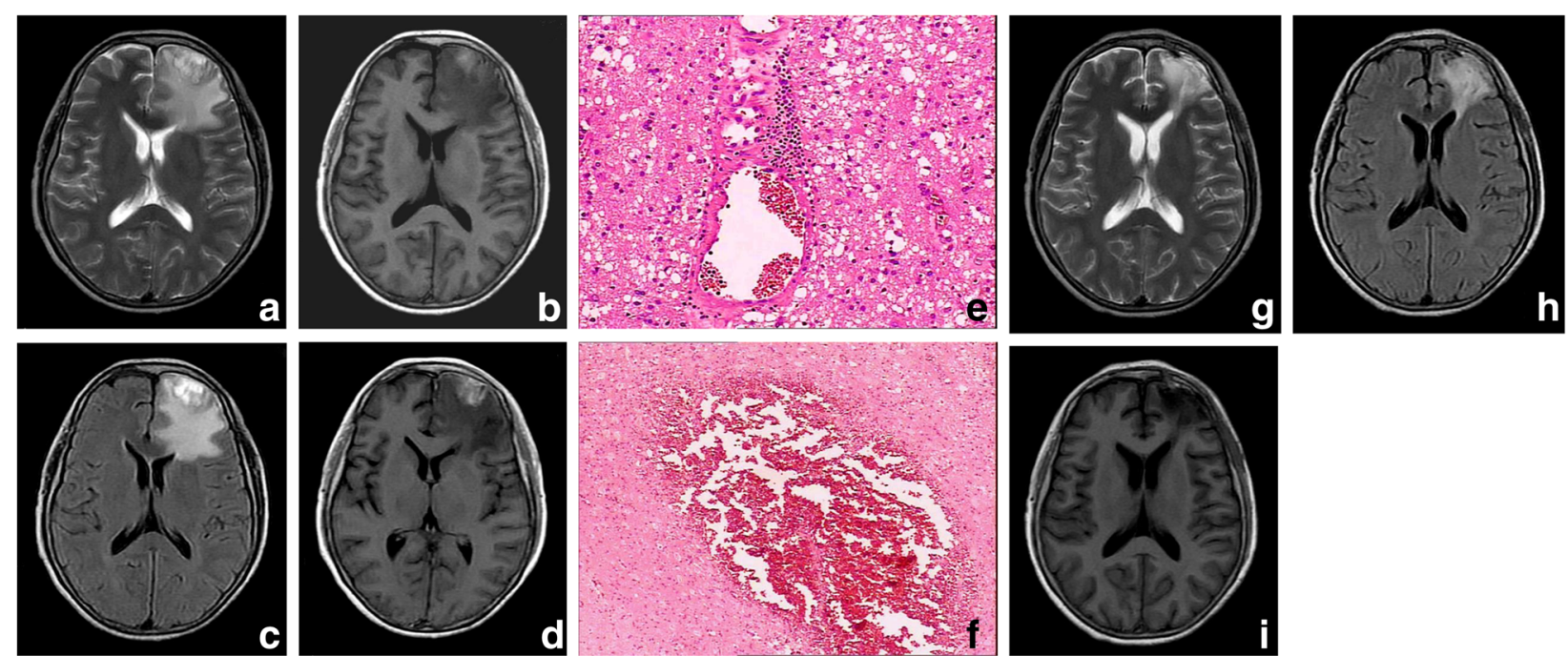

Fig. 1 a, b: Axial T2- and T1-weighted image shows a mixed slight hyperintense signal mass lesion in the left frontal lobe with severe brain edema. c: Increased signal of same lesion is noted on FLAIR image. d: Postcontrast T1-weighted image shows heterogeneous enhancement of ill-defined and dural-based lesion. e. Histological section of typical field showing perivascular infiltration of small lymphocytes, plasma cells, macrophages and histiocytes. Lymphocytes transmigrating to small vessel walls and intravascular IP tissue was noted (Hematoxylin and eosin staining, original magnification $\times 200)$. f. Histological section of some areas shows numerous thin-walled blood vessels, areas of congestion and hemorrhage (Hematoxylin and eosin staining, original magnification $\times 100$ ). g, h, i: Axial T2-weighted image, FLAIR image and T1weighted image six months after surgical section showed that there was no recurrence

department. During the operation, a brown and yellow granulomatous subcortical lesion was found to be of fibrous and ill-defined. The lesion contained multiple cysts, which attached to the hypervascularized and thickened dura, extended into the white matter. Finally, the lesion was completely resected under microsurgery. Histopathological examination of the specimen revealed a diffuse infiltration of gray matter and white matter by inflammatory cells consisting of mature lymphocytes, plasma cells, macrophages and histiocytes. There was no evidence of mitosis, anaplasia or necrosis. In some areas, lymphocytes were suspected to transmigrate to small vessel walls and intravascular IP tissue was found in the lesion (Fig. 1e). Numerous thin-walled blood vessels, areas of congestion and hemorrhage were also noted (Fig. 1f). Immunohistochemical staining was performed by antibodies against GFAP, vimentin, EMA, S-100, CD3, CD20, CD79, CD68, CD163 and CD34. Immunohistochemistry showed that lymphocytes were composed of a mixture of CD3, CD20, and CD79 positive cells. Immunohistochemical studies confirmed the presence of histiocytic and plasma cell populations. Many CD68 positive histiocytes and CD163 positive plasma cells in the involved brain tissue were noted, but they were negative for S-100, CD34, GFAP, vimentin and EMA. Few S-100 immunopositive cells were found in some area of hemorrhage. In short, histopathological findings further confirmed the diagnosis of inflammatory demyelinating disease.
The patient was in good condition with no neurologic deficits after surgical intervention. Low-dose steroid treatment with oral administration of methylprednisolone (40 mg, bid) was continued as well. A follow-up enhanced CT scans four weeks after surgery showed the mass lesion was absence and surrounding edema was much smaller. Repeat MR imaging three months after the corticosteroid therapy revealed the absence of gadolinium enhancement and a decrease in the size of edema region (Fig. 1g, h, i). There was no recurrence after 6 months.

\section{Discussion}

IP of the CNS is a pathologic term that describes a reactive, inflammatory nonmalignant phenomenon that clinically and radiographically mimics a neoplasm in the brain. It was originally described as a nonneoplastic process and reported under different names including: inflammatory myofibroblastic tumor, plasma cell granuloma, xanthomatous pseudotumor, pseudosarcomatous, fibromyxoid tumor and inflammatory myofibrohistiocytic proliferation in the literature subsequently $[7,8]$. IP primarily affecting the central nervous system is rare. In most cases, the etiology of IP often is uncertain although several subset lesions are inflammatory and associated with a variety of infectious agents and may be caused by autoimmune disorders [ 9 , 10]. In addition, demyelinating diseases presented with tumor-like lesions, such as multiple sclerosis, acute disseminated encephalomyelitis and myelinoclastic diffuse sclerosis had also been reported in the literature $[11,12]$. The image 
features of IP may be as below: First, most IP exhibited some degree of enhancement. Second, some IP contained some degree of infiltrative features such as bone erosion and invasion of surrounding tissues. As reported by Ginat et al, calcifications were indentified in the dural IP. Pathological examination can find inflammatory cells, such as lymphocytes, plasma cells, macrohpages, but evidence of mitosis, anplasia or necrosis.

Our case, by contrast, presented with no history of virus or bacteria infection. The mass lesion was located in the left frontal lobe, showed heterogeneous enhancement on MR images and infiltrative to the surrounding brain parenchyma with mass effect and severe brain edema. It resembled an atypical brain tumor in appearance of radiology and did not have a preference for periventricular area which is predilection for demyelinating diseases. Therefore, it did not quite match the diagnostic criteria of demyelinating lesion such as multiple sclerosis, acute disseminated encephalomyelitis or myelinoclastic diffuse sclerosis. As far as we know, such a case was conventionally termed tumorlike demyelinating lesions or monofocal acute inflammatory demyelination in the previous reports [13]. Actually, the present case was similar to the published cases with tumorlike demyelinating lesions and accompanied by meningeal involvement which was evidently demonstrated on MR images. We speculate that the same radiologic appearance may contribute to the serviceability of image diagnosis for tumor-like demyelinating lesion [14].

\section{Conclusion}

Tumor-like demyelinating lesion is a rare form of intracranial IP and the correct diagnosis mainly depends on the postsurgical histopathologic examinations due to the rarity [15]. On the other hand, immunohistochemical staining plays an important role in the differential diagnosis. Usually, CD34 positivity was considered as a typical distinguishment between the neoplastic and nonneoplastic groups. In this case, positive immunostaining confirmed the presence of lymphocytes (by CD3, CD20 and CD79), macrophages or plasma cells (by CD163, CD68), and meanwhile, negative immunostaining for S-100, GFAP, vimentin and EMA eliminated the diagnosis of neoplasm resembled such as plasmacytoma, lymphoma, lymphoplasma cell-rich meningioma, and histiocytosis. A final diagnosis of primary IP of tumorlike demyelinating lesion was made based on the histopathological features, immunohistochemical studies and radiographical manifestations.

For the treatment options of primary intracranial IP of tumor-like demyelinating lesion, there remains no consensus regarding schedules in the literature [16]. As is known to all, despite their aggressive appearance, they may respond well to steroid treatment. So, we suggest complete resection of the intracranial lesion and involved dura, if possible, followed by administration of short term oral prednisolone therapy will be good for preventing recurrence.

\section{Competing interests \\ The authors declare that they have no competing interests.}

\section{Authors' contribution}

YM participated in its design and coordination, and drafted the manuscript. $\mathrm{HM}$ and JM carried out the pathological examination. XC provided the image data and studied the imageology character of this case. GL performed the treatment, conceived of the study, and helped to draft the manuscript. All authors read and approved the final manuscript.

\section{Author details}

${ }^{1}$ Department of Neurosurgery, Zhejiang Provincial People's Hospital, Hangzhou, Zhejiang 310014, China. 'Department of Pathology, Zhejiang Provincial People's Hospital, Hangzhou, Zhejiang 310014, China. ${ }^{3}$ Department of radiology, Zhejiang Provincial People's Hospital, Hangzhou, Zhejiang 310014, China.

Received: 2 July 2015 Accepted: 20 October 2015

Published online: 18 February 2016

\section{References}

1. Bahadori M, Liebow AA. Plasma cell granulomas of the lung. Cancer. 1973; 31(1):191-208.

2. West SG, Pittman DL, Coggin JT. Intracranial plasma cell granuloma. Cancer. 1980;46(2):330-5.

3. Häusler M, Schaade L, Ramaekers VT, Doenges M, Heimann G, Sellhaus B. Inflammatory pseudotumors of the central nervous system: report of 3 cases and a literature review. Hum Pathol. 2003;34(1):253-62.

4. Saxena A, Sinha S, Tatke M. Intracranial plasma cell granuloma - a case report and review of the literature. Br J Neurosurg. 2000;14(5):492-5.

5. Gandhi RH, Li L, Qian J, Kuo YH. Intraventricular inflammatory pseudotumor: Report of two cases and review of the literature. Neuropathology. 2011; 31(4):446-54.

6. Ginat DT, Bokhari A, Bhatt S, Dogra V. Inflammatory pseudotumors of the head and neck in pathology-proven cases. J Neuroradiology. 2012;39(2):110-5.

7. Lui PC, Fan YS, Wong SS, Chan AN, Wong G, Chau TK, et al. Inflammatory pseudotumors of the central nervous system. Hum Pathol. 2009;40(11):1611-7.

8. Badalian-Very G, Vergilio JA, Degar BA, Rodriguez-Galindo C, Rollins BJ. Recent advances in the understanding of Langerhans cell histiocytosis. Br J Haematology. 2012;156(2):163-72.

9. Saab ST, Hornick JL, Fletcher CD, Olson SJ, Coffin CM. IgG4 plasma cells in inflammatory myofibroblastic tumor. Mod Pathol. 2011;24(2):606-12.

10. Gomez FP, Steelman AJ, Young CR, Welsh CJ. Hormone and immune system interactions in demyelinating disease. Horm Behav. 2013;63(2):315-21.

11. Caroli E, Salvati M, Ferrante L. Tumor-like multiple sclerosis: Report of four cases and literature review. Tumori. 2006;92(6):559-62.

12. Turatti M, Gajofatto A, Bianchi MR, Ferrari S, Monaco S, Benedetti MD. Benign course of tumour-like multiple sclerosis. Report of five cases and literature review. J Neurol Sci. 2013;324(2):156-62.

13. Kimura N, Kumamoto T, Hanaoka T, Hasama Y, Nakamura K, Okazaki T Monofocal large inflammatory demyelinating lesion, mimicking brain glioma. Clin Neurol Neurosurg. 2009;111(10):296-9.

14. Puntambekar P, Santhakumar S, Kupsky WJ, Tselis A, Mittal S. Primary intracranial plasma cell granulomas presenting as malignant neoplasms. J Neurooncol. 2012;106(7):327-37.

15. Vedeler CA, Farbu E, Mellgren SI. Chronic inflammatory demyelinating polyneuropathy. Acta Neurol Scand. 2013;127(Suppl 196):48-51.

16. Flannery T, Al-Sabah F, Bhangu J, Alderazi Y, Brett F, Pidgeon C. Treatment of subtotally resected intracranial plasma cell granuloma with steroids: a case report. Br J Neurosurg. 2007;21(5):501-3. 